Systematic review and meta-analysis

\section{Early mobilisation after transfemoral catheterisation is not associated with increased vascular incidents, and reduces back pain}

10.1136/eb-2014-101782

\section{Sek Ying Chair}

The Nethersole School of Nursing, The Chinese University of Hong Kong, Hong Kong, Hong Kong

Correspondence to: Dr Sek Ying Chair, 8/F, Esther Lee Building, The Nethersole School of Nursing, The Chinese University of Hong Kong, Shatin, Hong Kong, Hong Kong; sychair@cuhk.edu.hk

Commentary on: Mohammady $\mathbf{M}$, Heidari K, Akbari Sari A, et al. Early ambulation after diagnostic transfemoral catheterisation: a systematic review and meta-analysis. Int J Nurs Stud 2014; 51:39-50.

\section{Implications for practice and research}

- A shorter period of bed rest (2-3 h) after diagnostic transfemoral catheterisation did not increase the risk of vascular incidents. It did improve back pain and reduce urinary discomfort.

- Early ambulation implies a probable reduction in the cost of hospitalisation and nursing care.

\section{Context}

While bed rest has been shown to be beneficial in the period following diagnostic transfemoral catheterisation, accumulating evidence suggests a shorter bed rest period may be as effective at preventing vascular complications (eg, bleeding and haematoma) as a longer one. ${ }^{1}$ A prolonged duration of bed rest may also be associated with more discomfort, patient dissatisfaction, back pain, voiding problems and groin site pain. ${ }^{2}{ }^{3}$ No conclusion has been made regarding the optimal bed rest period because definitions of early and late ambulation vary considerably among studies. ${ }^{2-4}$ This review evaluated all known interventional studies assessing the effect of the duration of bed rest after transfemoral catheterisation on the incidence of vascular complications.

\section{Methods}

The systematic review identified relevant studies using multiple electronic databases. Studies were eligible if they were randomised controlled trials -studies which used a randomised method for subject allocation or quasi-randomised controlled trials-studies which used systematic methods such as case record number for subject allocation, patients were older than 18 years, and underwent cardiac or non-cardiac transfemoral diagnostic catheterisation. Primary outcomes included the incidence of haematoma at the puncture site and bleeding. The electronic search identified 2508 references, and 42 full texts were retrieved. Twenty studies with 4019 participants were included. Studies assessing bed rest duration ranging from 2 to $24 \mathrm{~h}$ were categorised into three groups according to length of the bed rest period.

\section{Findings}

The incidence of bleeding and haematoma, bruising, pseudoaneurysm and thrombus or arteriovenous fistula among patients having $2 \mathrm{~h}$ bed rest did not differ from those having $4 \mathrm{~h}$ bed rest. The difference in results remained not significant when early and late ambulation were defined differently (ie, 2-4 vs $6 \mathrm{~h}$ and $4-6$ vs $12-24 \mathrm{~h}$ ). The review also suggested that reduced bed rest might improve back pain and urinary discomfort.

\section{Commentary}

This paper addresses an important topic of nursing practice. Transfemoral catheterisation is a common procedure and the selected patient outcomes including vascular complications, general discomfort, back pain, urinary discomfort and patient satisfaction, are the most common complications to require nursing attention. Bed rest has been reported to be beneficial; however, suboptimal patient outcomes can also result from prolonged bed rest. Varying bed rest periods have been proposed, thus causing confusion in practice. In this review, the effect of a wide variation of bed rest duration ranging from 2 to $24 \mathrm{~h}$ was investigated.

Through synthesising the best available evidence, $2-3 \mathrm{~h}$ bed rest following transfemoral catheterisation can reasonably be recommended. The results of this review are congruent to a similar review published in 2008. ${ }^{1}$ The notion of early ambulation after transfemoral catheterisation, proposed by nurses and cardiologists for years, is now supported by accumulating empirical evidence. The review, which included studies conducted in different populations, provides grounds to change current nursing practice in many places across the world. Unfortunately, a randomised controlled trial evaluating similar outcomes with a sample size of 137 patients was not published early enough to be included in this review. ${ }^{3}$

With the development of catheters and devices, as well as the development of skills for catheterisation, a transradial approach is becoming more popular because of the lower incidence of major vascular complications, and due to early ambulation and rapid recovery after the procedure. ${ }^{5}$ However, evidence to inform care of patients after transradial catheterisation is still limited. Future studies in this area should be encouraged.

Competing interests None.

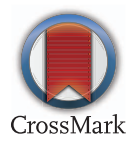

\section{References}

1. Chair SY, Fernandez R, Lui MHL, et al. The clinical effectiveness of length of bed rest for patients recovering from trans-femoral diagnostic cardiac catheterisation. Int J Evid Based Healthc 2008;6:352-90.

2. Chair SY, Thompson DR, Li SK. The effect of ambulation after cardiac catheterisation on patient outcomes. J Clin Nurs 2007;16:212-14.

3. Chair SY, Yu M, Choi KC, et al. Effect of early ambulation after transfemoral cardiac catheterisation in Hong Kong: a single blinded randomized controlled trial. Anadolu Kardiyol Derg 2012;12:222-30.

4. Dowling K, Todd D, Siskin G, et al. Early ambulation after diagnostic angiography using 4-F catheters and sheaths: a feasibility study. J Endovasc Ther 2002;9:618-21.

5. Cheng KY, Chair SY, Choi KC. Access site complication and puncture site pain following transradial coronary procedures: a correlation study. Int J Nurs Stud 2013;50:1304-13. 